\title{
EXPERIENCIAS
}

\section{Prevención del comportamiento delictivo en la comunidad}

\section{The prevention of the criminal behavior in the community}

\section{Carmen ORTE SOCÍAS 1 Luis BALLESTER BRAGE ${ }^{2}$}

\section{RESUMEN}

La heterogeneidad de la población escolar y de los problemas de comportamiento tanto individuales y grupales como de convivencia en los centros educativos, y en determinados territorios de influencia del mismo, hacen necesario el desarrollo de programas de prevención y de intervención educativa que estén basados en modelos que posibiliten el análisis de los problemas y su intervención con los actores implicados dentro y fuera del sistema educativo. Modelos que tengan en cuenta los distintos subsistemas que integran el sistema escolar en un centro educativo y en un territorio concreto. Se trata de posibilitar el desarrollo de intervenciones generadas con los propios implicados. El programa de intervención comunitaria dirigido a población escolarizada de 12 a 18 años de edad al que se hace referencia en este artículo llevado a cabo en Baleares, se diseñó y ejecutó con ese objetivo. El programa se llevó a cabo en algunos municipios de la Comunidad Autónoma de las Islas Baleares contando con los diferentes actores y recursos implicados en cada uno de los territorios en los que se ubicaban los distintos centros educativos en los que tuvo lugar la intervención, desde una perspectiva comunitaria de trabajo en red

\section{PALABRAS CLAVE}

Prevención, Comportamiento antisocial, Conflicto escolar, Trabajo en red, Intervención comunitaria.

\footnotetext{
${ }^{1}$ Catedrática de Universidad. Departamento de Pedagogía y Didácticas Específicas. Campus UIB.

2 Titular de Universidad. Departamento de Pedagogia y Didácticas Específicas. Campus UIB
} 
Prevención del comportamiento delictivo en la comunidad

\section{ABSTRACT}

The heterogeneity of the student population, of behavior problems at individual or group level and of living-together problems makes educational prevention and intervention programs necessary. These programs need to be based on models that allow problem analysis and intervention with the agents who are involved both inside and outside the educational system. These models need to take into account the various subsystems making up a school system in a particular center or region. The target of the community intervention program addressed in this paper was the school population of the Balearic Islands, aged between 12 and 18 years old. The program was applied in a number of towns. The intervention was carried out from a web-based work community view.

\section{KEY WORDS}

Prevention, Antisocial behaviour, Scholar conflict, Net work, Communitarian intervention. 
En este artículo abordamos la prevención del comportamiento delictivo en la comunidad, a partir de la experiencia en el Programa de Intervención Comunitaria llevado cabo en algunos municipios de la Comunidad Autónoma de las Islas Baleares (Varios autores, 1999). Se trata de un programa diseñado para la intervención y prevención en los problemas de conflicto juvenil de los jóvenes de 12 a 18 años. De acuerdo con la edad de los jóvenes a los que se dirige el programa, los contextos que se abordan de forma particular son, la comunidad educativa, el alumnado, las familias, el profesorado, y los grupos de iguales.

Los aspectos a los que vamos a hacer referencia son los que siguen:

1. Cómo entendemos el conflicto;

2. Cómo entendemos el papel de la intervención preventiva $\mathrm{y}$

3. Cómo abordar la intervención preventiva desde una perspectiva comunitaria basada en el trabajo en red a partir de Comisiones Técnicas.

\section{El conflicto y la violencia desde una perspectiva comunitaria}

El término conflicto puede referirse a diferentes significados no necesariamente unidos a connotaciones negativas. No obstante, en general y en el lenguaje cotidiano, suele utilizarse con dicha connotación, unida a aspectos de no-cooperación, comunicación negativa, intolerancia, expresión negativa de las emociones y utilización de estrategias de resolución de conflictos de tipo negativo, agresivo y/o pasivo, que se producen de forma cotidiana dentro de una comunidad de vida y de relación (Barroso, Buxarrais y Orte, 2002).
Al respecto, apenas disponemos de modelos y experiencias cotidianas positivas. Buena parte de las actitudes que impregnan nuestra cultura de la resolución de diferencias y desencuentros, tienen que ver con el todo o nada, con la fuerza y la debilidad, y con el ganar o perder. Los media, como reflejo del acontecer en diversos planos: político, económico, cultural, educativo, relacional, etc., reflejan día tras día, la importancia de este tipo de actitudes de partida, que fomentan esta visión negativa: "lo que yo quiero" "mis metas" "mis objetivos", "cueste lo que cueste" .... Frente a este tipo de actitudes en las que alguien debe ganar y alguien debe perder, en las relaciones personales y sociales, sólo cabe, pues, o la misma actitud combativa de "ganar o perder" 0 , la de aceptar la derrota individual "pierdo, ganas" o la colectiva, "destruir frente a construir" en la que todos pierdan.

Los conflictos que se producen en una comunidad determinada, la escolar, por ejemplo, pueden referirse a varias conductas y situaciones que tienen lugar en la misma, entre otras: problemas genéricos de disrupción en las aulas o en otros lugares de la escuela como pasillos, patio de recreo, comedor, baños, zonas de acceso al recinto etc. Se trata de conflictos -generalmente percibidos como amenazantes-, entre alumnos y profesorado, agresión, intimidación y abuso entre alumnos, daños a la propiedad de los alumnos, de los profesores o de la institución escolar..... etc. El concepto, no se refiere sólo a un conflicto mal resuelto de intereses entre las diversas personas y grupos que forman parte del entorno escolar. Ya que ello no implica necesariamente una respuesta negativa, violenta y aversiva, al contrario, puede ser una ocasión para aprender a dialogar, a negociar, a pactar o a cooperar. Se refiere más bien, a una forma muy concreta de relación en la que se incluye la idea de 
que se trata de situaciones conflictivas que cursan con violencia, es decir, se trata de comportamientos en los que se utiliza el poder sobre el otro de forma prepotente, asimétrica y negativa (Orte, 2003, 2006).

El nivel de conflictividad -entendida como agresividad y violencia en las relaciones entre sus miembros- que presenta una institución, trasciende la dimensión individual que pueda estar presente en un momento determinado cuando surge un episodio agresivo en el que está implicado un joven o un grupo de jóvenes concreto, y adquiere una dimensión más contextual, en la que los sistemas implicados en el mismo son varios y en el que los aspectos de tipo sociocultural adquieren mayor protagonismo.

Se requiere de un análisis profundo, contextual y no estático de las "personas y situaciones" generadoras de conflicto. En cualquier contexto la convivencia diaria produce desacuerdos y conflictos, la cuestión no es pues que haya conflictos, sino las respuestas que se den a los mismos; así, los conflictos pueden producir disrupción y disfunción, respuestas autoritarias, represoras 0 violentas, sentimientos de ofensa y humillación, lo importante son las respuestas que se dan al mismo. La visión del conflicto de una forma positiva en una institución concreta, acompaña la generación de nuevas respuestas. Dichas respuestas, por ejemplo, pueden ayudar a mejorar el clima organizativo, las rutinas diarias, las relaciones entre diferentes grupos, crear nuevos sistemas organizativos, etc. En definitiva, el conflicto debe servir para un propósito útil en el que los distintos grupos que conforman la realidad de una institución concreta, tienen que llevar a cabo un esfuerzo importante de reflexión compartida y consensuada sobre el modelo de persona a la que se dirigen, y sobre el papel y funciones que corresponde llevar a cabo de acuerdo a los cambios que se han producido en diversos niveles en la sociedad.

De acuerdo con ello, es evidente que el fracaso en ningún modo tiene una dimensión individual, aunque pueda haber aspectos del propio individuo que contribuyan a elaborar un determinado tipo de respuesta. Se tiene que hablar del fracaso de la administración, de la institución educativa, del profesorado, de las familias, de las redes y de los recursos sociales y culturales, de las metodologias y didácticas, de la propia cultura social que tiene dificultades para integrar, para afrontar las necesidades que puede plantear un individuo, un grupo, una familia concreta. Los conflictos que cursan de forma negativa y que se resuelven de forma violenta se forman a lo largo de procesos dilatados, no aparecen de pronto ni por azar (Orte, Ballester, Oliver, 2002).

Si entendemos el conflicto como fracaso, es decir, como resultado de la acumulación de situaciones problemáticas no resueltas de forma satisfactoria, parte del conflicto se explica por el choque cultural (por ejemplo, escuela-grupos sociales). Es evidente que se impone utilizar medidas como, entre otras, el aprendizaje institucional de estrategias socio-cognoscitivas para la resolución de conflictos; la creación de estructuras flexibles de acompañamiento social y educativo; estrategias contextualizadas en la intervención con los padres y con la familia; estrategias de visitas en el hogar etc. Dada la heterogeneidad de la población y del contexto escolar social y cultural, hay que tener en cuenta que cada centro escolar, cada territorio, genera sus fracasos y tiene su propio tipo de conflictos. Se han de tener presentes las relaciones con el territorio-entorno (Orte, Ballester, Oliver, 2002). 
Desde este punto de vista, pues, tanto el conflicto como la violencia juvenil pueder resultar sinónimos en un determinado contexto. En cualquier caso, su complejidad incluye numerosos factores de riesgo de tipo individual, familiar y social. Esta complejidad presenta

\section{La intervención preventiva desde una perspectiva comunitaria}

De acuerdo con esta filosofia, el programa de prevención y abordaje de las situaciones de conflicto juvenil (gráfico número 1) contempla dos grandes

\section{Gráfico 1. Esquema Programa de Prevención y Abordaje de las Situaciones de conflicto Juvenil}

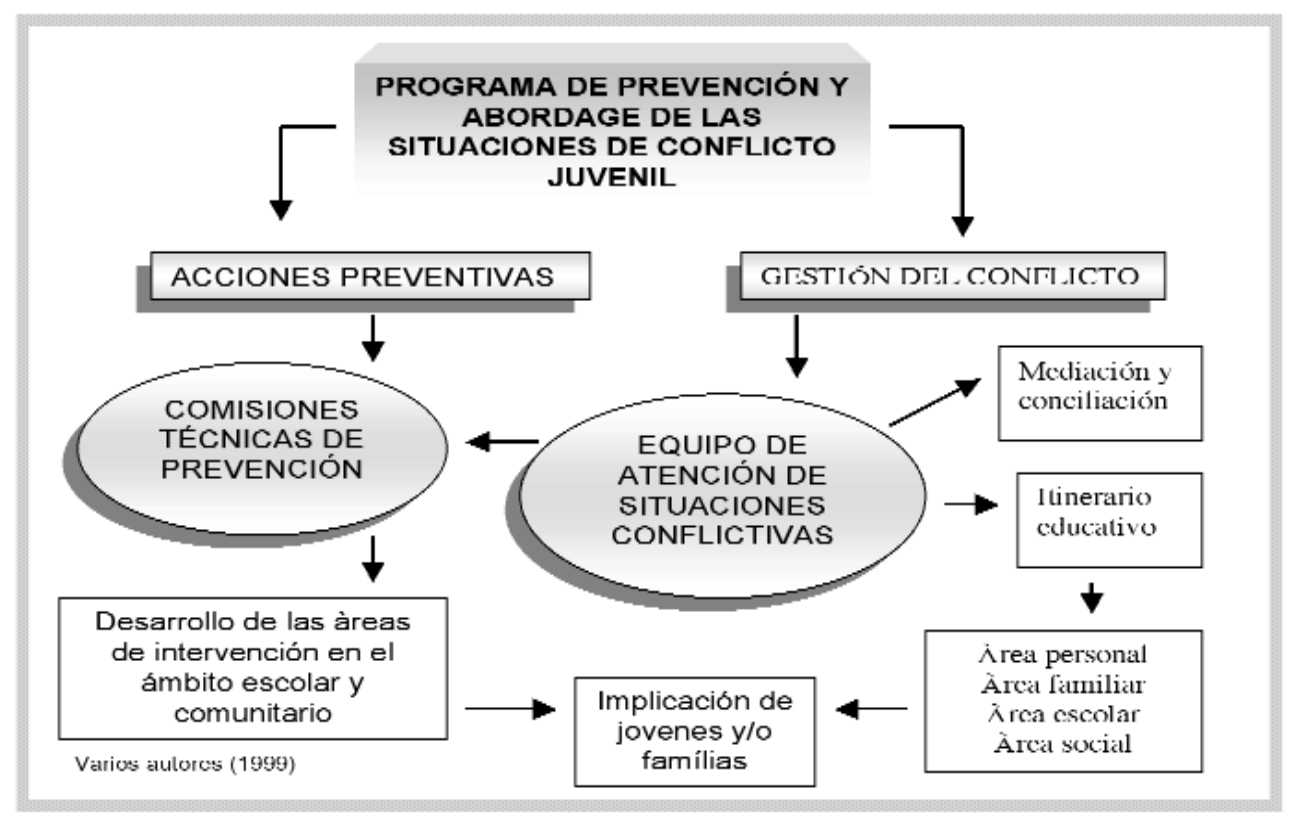

numerosos retos para la intervención, y especialmente para la intervención preventiva. En este contexto, el enfoque comunitario de la intervención, posibilita la responsabilidad compartida de las instituciones, permite la participación interinstitucional y la generación, creación y puesta en marcha de alternativas. Además, favorece las acciones sumativas y coordinadas de las instituciones, que confluyen en un determinado territorio y también la de aquellas que tienen responsabilidad sobre el problema y/o sobre el individuo o grupo en cuestión. acciones relacionadas: por una parte, las acciones preventivas territoriales, en las que la acción preventiva se contextualiza a partir de las Comisiones Técnicas de Prevención a las que hacemos referencia en este documento, cuyo objetivo y objeto de acción es el ámbito escolar y el comunitario.

Las Comisiones tienen una composición representativa y un carácter descentralizado a partir del criterio de insularidad y municipalización. Están constituidas por representantes de los recursos educativos y sociales relaciona- 
dos con los jóvenes de 12 a 18 años. Los recursos socioeducativos y culturales han sido, preferentemente los siguientes: Servicios Sociales de Atención Primaria, Servicios Educativos Municipales, Centros de Atención Secundaria, Servicios de Protección de Menores, Policia Local, AMPAS, y otros colectivos que se han ido incorporando a algunas sesiones cuando ha sido necesario. $\mathrm{Su}$ composición oscila entre 8 y 12 personas. El municipio determina, en cada caso, la persona que preside la Comisión. Por su parte, las funciones de Secretaría las lleva a cabo un técnico educador o educadora social, del Equipo de Atención a las Situaciones de Conflicto Juvenil de la Dirección General de Menores y Familia. Dicho equipo, por su parte, es también una pieza central del trabajo de gestión del conflicto que se lleva a cabo desde la perspectiva comunitaria, con el resto de instituciones, además de la escolar, que integran un determinado territorio.

\section{Abordar la intervención preventiva desde una perspectiva comunitaria basada en el trabajo en red a partir de Comisiones Técnicas}

Los objetivos de Comisiones Técnicas de Prevención del Conflicto Juvenil de acuerdo a la actividad llevada a cabo se han centrado principalmente en los siguientes (Varios autores, 2003; Orte et als. 2003):

- La valoración de las necesidades que presentan las diferentes instituciones y/o servicios que desarrollan una acción socioeducativa dirigida a jóvenes entre 12 y 18 años de la zona de influencia de la Comisión.
Para llevar a cabo la valoración de las necesidades, las Comisiones han utilizado el sistema de diagnóstico DAFO (Ballester, 2001). Dicho sistema, permite organizar y comprender la información disponible. Se presta atención a cuatro aspectos fundamentales: Debilidades, Amenazas, Fortalezas y Oportunidades (cuadro número 1), en relación a la situación de los problemas y de los recursos de la, en este caso, zona de influencia analizada.

La combinación de estos cuatro aspectos posibilitan las estrategias de acción (Oliver et als. 2003): Así, la combinación de Debilidades/Amenazas, indica que hay que llevar a cabo estrategias de atención preferente, acciones urgentes. La combinación de Debilidades/Oportunidades indica que se deben de llevar a cabo estrategias de reorientación , aprovechando las oportunidades de que se dispone en ese momento. Por su parte, la combinación de Fortalezas/Amenazas, señala la importancia de llevar a cabo estrategias preventivas para adelantarse a las amenazas; y, la combinación de Fortalezas/Oportunidades, indica estrategias de calidad de vida, la necesidad de aumentar los aspectos positivos ya presentes.

La aplicación del diagnóstico DAFO para el análisis de necesidades, se llevó a cabo tras una primera fase formativa, en la que se explicaba la técnica por parte de un miembro del Equipo Asesor y Evaluador del Programa ${ }^{1}$. En una segunda fase, se recogian las aportaciones del debate grupal, resultado de la aplicación del DAFO por cada una de las instituciones representadas en la Comisión. La selección de prioridades

\footnotetext{
${ }^{1}$ Un equipo mixto de profesorado de la Universidad Illes Balears y de la Universidad de Barcelona.
} 
Cuadro 1. Esquema Diagnóstico DAFO

\begin{tabular}{|c|c|c|}
\hline \multirow{4}{*}{$\begin{array}{c}\text { Esquema para el Análisis } \\
\text { DAFO }\end{array}$} & $\begin{array}{c}\text { Debilidades } \\
\text { (aspectos ncgativos quc } \\
\text { debieran modificarse) }\end{array}$ & $\begin{array}{c}\text { Situaciones actuales y } \\
\text { procesos actuales } \\
\text { Actual e Interno }\end{array}$ \\
\hline & $\begin{array}{c}\text { Amenazas } \\
\text { (riesgos potenciales que } \\
\text { debicran prevenirsc) }\end{array}$ & $\begin{array}{c}\text { Procesos que pucden darsc } \\
\text { cn un futuro y que gencran } \\
\text { obstáculos, limitaciones, } \\
\text { clc. } \\
\text { Potcncial o Actual y } \\
\text { externo }\end{array}$ \\
\hline & $\begin{array}{c}\text { Fortalezas } \\
\text { (aspcctos positivos quc } \\
\text { debicran mantencrsc o } \\
\text { reforzarse) }\end{array}$ & $\begin{array}{l}\text { Situaciones actuales y } \\
\text { procesos actuales }\end{array}$ \\
\hline & $\begin{array}{c}\text { Oportunidades } \\
\text { (capacidades y recursos } \\
\text { potenciales que se debieran } \\
\text { aprovechar) }\end{array}$ & $\begin{array}{c}\text { Factorcs quc hay quc } \\
\text { aprovechar para generar } \\
\text { proccsos positivos ¿por qué } \\
\text { cl momcnto actual cs cl } \\
\text { más adecuado? } \\
\text { Potcncial o Actual y } \\
\text { externo }\end{array}$ \\
\hline
\end{tabular}

(cuadro número 2), al final de cada sesión, supuso la aplicación de técnicas complementarias al análisis DAFO que facilitaron el desarrollo y ampliación de su alcance.
Después de identificar los aspectos sobre los que actuar y las acciones a desarrollar, la evaluación de las prioridades se lleva a cabo en base a los siguientes criterios: 
Cuadro 2. Viabilidad del Proyecto en relación a los recursos

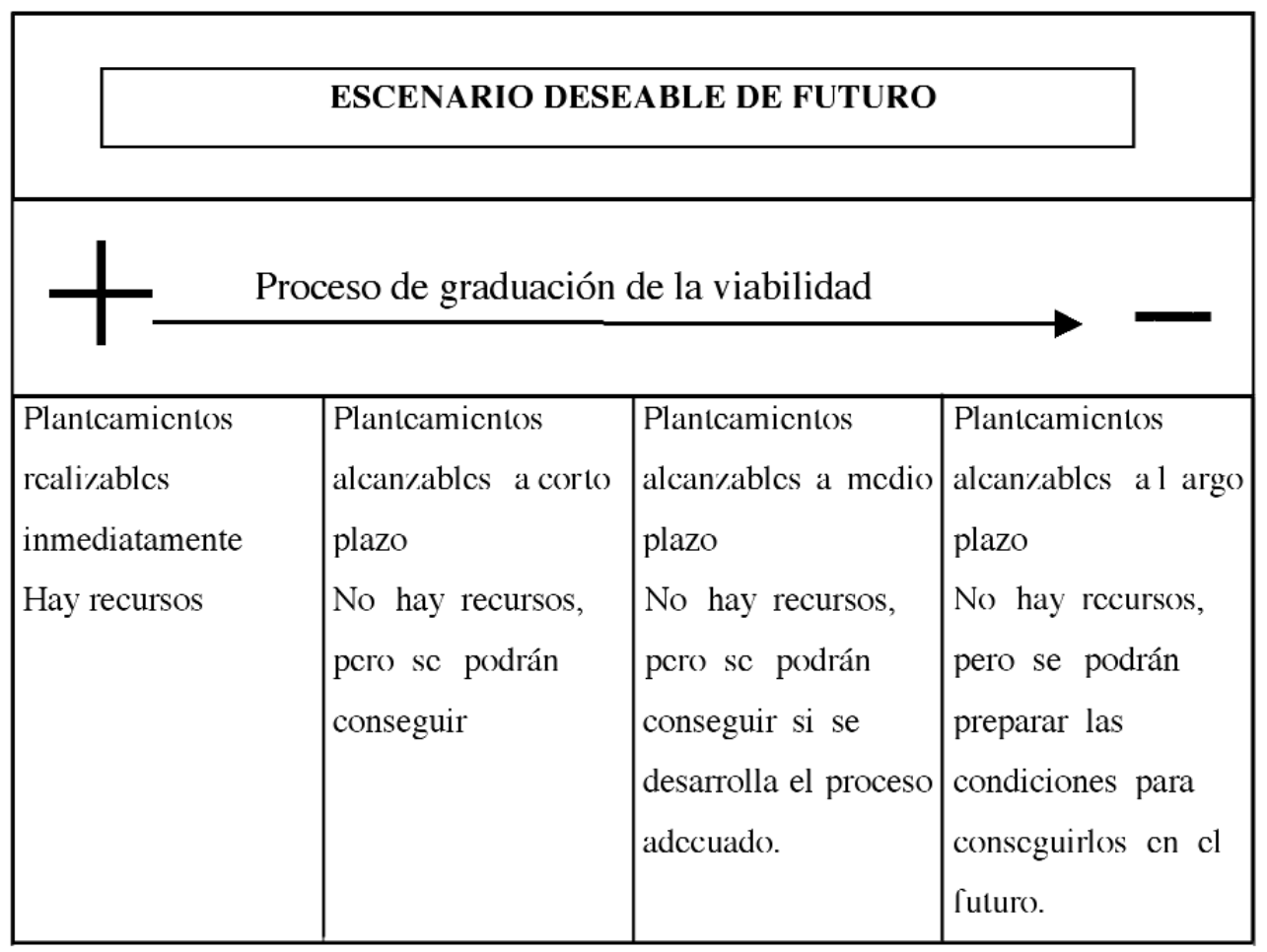

1. Importancia: A cuántas personas afecta, con que intensidad, etc.

2. Recursos y coste: Analizar si se dispone de recursos suficientes, si se pueden conseguir, si tiene un coste asumible en este momento 0 , más adelante, si el coste en relación al cambio que se producirá es asumible o no, etc.

3. Capacidad técnica: $\mathrm{Si}$ sabemos lo que hay que hacer, si conocemos otras experiencias realizadas y podemos controlar el error de los demás, etc.

4. Posibilidad de cambio: Que se conseguirá o qué se puede conseguir.
Para la asignación de valoraciones a cada grupo de criterios, se utilizó una puntuación que representa la prioridad (desde 0 , indeciso, no podemos valorarlo; hasta 3 , totalmente de acuerdo). Por ejemplo, si uno de los aspectos sobre los que el grupo ha considerado que se ha de actuar es "Modificar la falta de alternativas de ocio", y las acciones posibles para ello son:

1. Desarrollar alguna iniciativa de ocio organizada y adaptada a los jovenes de estas edades.

a) Actividades deportivas (futbol, balonmano, etc.)

b) Actividades culturales (teatro, cine, creación musical, excursiones, etc.). 


\begin{tabular}{|c|c|c|c|c|}
\hline Importancia & Recursos & Capacidad técnica & Cambio posible & Media \\
\hline 3 & 1 & 3 & 3 & 2,5 \\
\hline
\end{tabular}

c Actividades lúdicas y otros (juegos, baile, etc.).

Las puntuaciones podrían ser:

La valoración alcanza una puntuación muy alta en relación a la puntuación total, y las puntuaciones individuales indican una alta posibilidad y viabilidad de la misma. Con esta metodología, se consigue establecer un consenso bastante generalizado en relación a los aspectos de la realidad social del municipio sobre los que incidir para prevenir e influir de forma positiva sobre la problemática del conflicto juvenil así como sobre el orden en que conviene generar actuaciones o proyectos.

La utilización de este sistema dentro de la estructura de la Comisión Técnica ha permitido dar respuesta a los principales aspectos de la planificación y ejecución de la intervención preventiva (Thornton et als., 2001):

- Identificar a los individuos o grupos más afectados por el problema con mayor frecuencia.

- Disponer de argumentos para la intervención dirigidos a las instituciones y/o grupos implicados en el problema.

- Recoger datos cuantitativos y cualitativos en relación al problema, incluyendo datos de opinión de las personas $\mathrm{y} / \mathrm{o}$ grupos con alguna relación o responsabilidad en el mismo (los propios jóvenes, los profesores, equipos directivos, asociaciones de alumnos, de padres, de voluntarios, de vecinos, respon- sables de educación y bienestar, etc.).

- Seleccionar el grupo de intervención a partir de estos datos, tomando en consideración que el grupo de referencia del proyecto es el colectivo de 12-18 años Los datos recopilados han ayudado a las Comisiones Técnicas a tomar decisiones sobre el grupo hacia el cual orientar la intervención. De acuerdo con el análisis de las Actas de las Comisiones (2000-2002), el grupo de elección más importante han sido los niños y adolescentes de 14-16 años que exhiben conductas de alto riesgo como el abandono escolar (alumnos absentistas habituales y crónicos) o conflictos contra la norma, principalmente en el contexto escolar.

- Establecer metas y objetivos de intervención.

$\mathrm{Al}$ respecto de esta cuestión indicar que, aunque la actividad de las Comisiones Técnicas ha sido diversa dependiendo de las características y composición de la Comisión y del territorio de influencia. Un grupo importante de Comisiones ha delimitado sus metas y objetivos de intervención a la población 14-16 años. La mayor parte de las Comisiones ha centrado sus prioridades en este grupo de población. Las finalidades han sido varias, entre otras: mantener o recuperar a este grupo de jóvenes/alumnos y alumnas, para el sistema educativo; elaborar experiencias alternativas a la escolarización reglada; priorizar 
alternativas ligadas a la escolarización ordinaria incluyendo contenidos formativos prelaborales.

- Seleccionar la intervención más apropiada:

Las Comisiones han desarrollado una actividad muy importante a la hora de generar alternativas socioeducativas para responder a este colectivo. Las soluciones más ampliamente desarrolladas, inciden sobre aquellas propuestas que incluyen la idea de escolarización compartida (dentro o fuera del aula). Agrupaciones de jóvenes en talleres con alto contenido prelaboral y con responsabilidad compartida entre instituciones educativas y otras (Ayuntamientos, Organizaciones no Gubernamentales, etc.).

Las pautas para seleccionar una intervención de entre todas las posibles ha tenido que ver con las características del territorio y también de los participantes. Por otra parte, y teniendo en cuenta la estructura de la Comisión Técnica y el flujo informativo intra e inter comisiones, se ha posibilitado el poder sacar provecho de la actividad de los demás.

La mayoría de las intervenciones que se han desarrollado o se están desarrollando, incluyen el diseño de intervenciones múltiples y estrategias complementarias. Por citar algunas de ellas muy importantes en el diseño de estrategias preventivas de la violencia juvenil:

En primer lugar el tema de la intervención con la familia se ha impuesto como referencia en la mayoría de debates, siendo en alguna de las Comisiones objeto y meta de intervención de forma directa 0 indirecta. Así y dentro de las propuestas de escolarización compartida surge como meta el diseñar también estrategias para alcanzar a las familias "fuera del alcance" por motivos ligados tanto a aspectos funcionales del centro como a aspectos funcionales de las familias. Mediante propuestas estratégicas como el ajuste de los horarios de tutoría de los centros, la elaboración de tutorias familiares personalizadas o las visitas al hogar familiar. Otras propuestas que implican a la familia incluyen aspectos formativos algo más comprometidos como puede ser la formación en habilidades parentales de estas familias (Orte et als., 2006). Un segundo ámbito de interés han sido las estrategias de trabajo de tipo socio-cognoscitivo o la incorporaciòn de estrategias para resolver situaciones sociales problemáticas, que inciden en las conductas de violencia juvenil, dentro del curriculum de estos programas.

- Implicación de la Comunidad

Un aspecto a tener en cuenta en el desarrollo de las actividad llevada a cabo por las Comisiones ha sido la implicación de la Comunidad, mediante la implicación de los representantes de sus instituciones. Ello favorece la cohesión interna, el desarrollo del sentimiento de pertenencia y la obtención de recursos.

- Coordinar esfuerzos:

El trabajo que se puede y se ha llevado a cabo desde las Comisiones. permite aunar esfuerzos frente a situaciones y grupos que a menudo se solapan en las intervenciones de las diversas instituciones 
de una comunidad. Favorece una opinión más homogénea en lo que se refiere al análisis de los problemas de un territorio o de un grupo concreto, que pueden estar ligados a la violencia juvenil. Posibilita el diseño de actividades preventivas desde una posición de corresponsabilidad social y profesional.

- La formación de un equipo interinstitucional con cierta estabilidad, y capacidad para valorar y priorizar las necesidades de su zona de influencia.

Como se ha comentado, es importante señalar el trabajo llevado a cabo desde el espacio de las Comisiones Técnicas, por parte de diversas instituciones educativas y sociales, como por ejemplo, por citar algunas: responsables técnicos de departamentos municipales de bienestar social, educadores sociales municipales, asesores de educación, coordinadores de talleres ocupacionales, orientadores, jefes de estudios y tutores de centros educativos, mediadores interculturales, representantes de la policía local y judicial, técnicos de infancia y familia, trabajadores sociales municipales, representantes de asociaciones de alumnos, etc.

Lógicamente, la propia dinámica interna de las Comisiones y el número de participantes genera dinámicas de trabajo en subcomisiones que enriquecen y agilizan el trabajo de la Comisión.

- El desarrollo de una tarea orientada a aumentar la toma de perspectiva de los profesionales y/o de las instituciones en su conjunto, en torno a la importancia de las acciones preventivas, relacionadas con posibles situaciones de conflicto que pueden traer como consecuencia, conductas de violencia.

El espacio creado a partir de las reuniones de las Comisiones posibilita una visión común del conflicto juvenil y de los problemas y soluciones que pueden llevarse a cabo de forma conjunta en un territorio concreto. También de las dificultades que cada centro y/o institución enfrenta para poder solucionarlas. En la mayoría de Comisiones ello aporta como resultado, la realización de proyectos comunes que gozan de amplio consenso por parte de sus miembros.

- La propuesta de alternativas realistas, de acuerdo con los recursos disponibles y con los que puedan proponerse, a los problemas priorizados de la zona.

Este objetivo resume de forma concreta tanto los procesos, como las actividades llevadas a cabo a lo largo del propio ciclo de la Comisión Técnica a la que hemos hecho referencia anteriormente.

\section{Conclusiones}

En estos momentos más que nunca, la heterogeneidad del alumnado de los centros y la diversidad de situaciones y necesidades de la comunidad en la que se inscriben, reclaman la necesidad de que los centros sean verdaderos recursos de la comunidad, que sean flexibles y sean algo más que un edificio que forma parte del paisaje de un territorio determinado. Que estén insertos en la comunidad a partir del trabajo socioeducativo que se lleva a cabo dentro y fuera de los mismos, es decir de acciones que incluyan una visión multi- 
dimensional de la socialización y de la educación. Ya hemos comentado la importancia del diseño de estrategias preventivas de tipo comunitario, pero no queremos finalizar sin señalar que es importante que la Administración educativa lleve a cabo proyectos conjuntos con otras Administraciones, en la línea ya iniciada, como por ejemplo la de Bienestar Social. También, que empiece a profundizar en el logro de una mayor implicación de la Administración Local en los proyectos socioeducativos que se lleven a cabo en los centros. Asi por ejemplo, tratamiento del conflicto desde diversas perspectivas: absentismo escolar, tratamiento de la diversidad social y cultural, implicación de las familias de forma profunda en la elaboración, desarrollo y ejecución de los proyectos educativos de los centros, etc., e implicando a otros agentes que puedan estimular y colaborar en la puesta en marcha de acciones socioeducativas adecuadas a las necesidades del alumnado de los centros. La obligatoriedad de elaborar planes de convivencia por parte de los centros educativos puede dar un gran impulso a estas acciones colectivas.
Un planteamiento de este tipo, requiere estructuras flexibles y diversidad de respuestas ante situaciones de dificultad, que permitan por ejemplo, realizar adaptaciones curriculares de forma habitual, incluir sistemas de refuerzo, llevar a cabo el seguimiento y la tutoría personalizada de alumnado y familias, poner en marcha estrategias de acompañamiento socializador en los adolescentes, agrupaciones flexibles de alumnos (no segregadoras), sistemas de negociación y mediación ante los conflictos, sistemas de reparación, sistemas participativos de reacción ante los conflictos, en definitiva, todas aquellas respuestas que faciliten el tratamiento de la diversidad desde una perspectiva inclusiva y no segregadora. Todas aquellas iniciativas que sean oportunas, como resultado del análisis de las necesidades que presenten, en este caso los jóvenes de 12 a 18 años, derivadas, de las dificultades que aparecen a lo largo de su desarrollo, tanto a nivel personal, como en los diversos contextos en los que se inscriben. Así, familia, escuela y comunidad forman un todo en el que lo que se pretende es fomentar trayectorias en las que el obtener éxito sea viable. 


\section{BIBLIOGRAFÍA}

Ballester, L. (2001). Anàlisi de necessitats i prioritats d'actuació de les Comissions. En, Programa de Prevención y Abordaje de las Situaciones de Conflicto Juvenil. Palma de Mallorca: UIB ( Documentación interna).

Barroso, C., Buxarrais, R., Orte, C. (2002). La acción educativa en el medio social. En, P. Ortega (coord.). Conflicto, violencia y educación (págs. 157205). Murcia: Cajamurcia.

Oliver, J.L., Ballester,, L., Orte, C. (2003). El análisis "DAFO" aplicado a la evaluación de necesidades en un contexto comunitario: Una base para la actuación municipal en relación a programas de prevención del conflicto. En, I Congreso Internacional. Convivencia Juvenil: Repensar El Conflicto. Palma de Mallorca, febrero, UIB/Direcció General de Menors i Familia.

Orte, C., Ballester, L., Oliver, J.L. (2002). Estrategias para la resolución de conflictos. Una propuesta de intervención desde el contexto comunitario. En, X Congreso de Formación del Profesorado: La formación del profesorado ante el fenómeno de la violencia y convivencia escolares. Cuenca, junio.

Orte, C., Ballester, L., Oliver, J.L., (2003). Estrategias de prevención de conflictos: Análisis del trabajo en red desde una perspectiva comunitaria. En, I Congreso Internacional. Convivencia Juvenil: Repensar El Conflicto. Palma de Mallorca, febrero, UIB/Direcció General de Menors i Familia.

Orte, C. (2006). Nuevas perspectivas sobre la violencia y el bullying escolar. Panorama Social, 3, 27-41

Orte, C., Touza, C., Ballester, Ll., March, M.X. (2006). Evaluación de resultados y de proceso del programa de competencia familiar. Revista Proyecto, $58,80-83$.

Thornton, T., Craft, C.A., Dahlberg, L.L., Lynch, B.S., Baer, K. (eds.). (2001). Practicas óptimas para la prevención de la violencia juvenil. (págs. 4-37) Atlanta: Departament of Health and Human Services.

Varios autores (1999). Programa de prevención y abordaje de las situaciones de conflicto juvenil en la CAIB 1999-2002. Palma de Mallorca: Govern Balear.

Varios autores (2003). Memoria del programa de prevención y abordaje de las situaciones de conflicto juvenil en la CAIB 2002. Palma de Mallorca: Govern Balear. 